\title{
ESTADO ACTUAL DEL CONOCIMIENTO DE LA FAMILIA STERCORARIIDAE EN EL EXTREMO SUR DE CHILE
}

\author{
STATE OF KNOWLEDGE ABOUT FAMILY STERCORARIDAE IN SOUTHERN CHILE
}

\author{
Ricardo Soto ${ }^{1}$, Víctor H. Ruiz R. ${ }^{1}$ \& E. Vega-Roman ${ }^{1}$
}

\begin{abstract}
Chilean skua belongs to a relatively low studied family, and the investigations on this group involve mostly sightings during expeditions for birds in extreme southern of Chile. They are also called "salteadores" (robbers) because of their opportunistic costumes; usually, they are described as big, aggressive and scavenger sea gulls. The term "skua" comes from the faroese "skügver", which means "feather tuft" and from Skúvoy island in the Feroe Islands archipielago, in Norwegin Sea.

In Chile there are six species: Stercorarius chilensis Bonaparte 1957, Stercorarius longicaudus Vieillot 1819, Stercorarius antarcticus (Lesson, 1831), Stercorarius maccormicki (Saunders, 1893), Stercorarius parasiticus (Linneo, 1758) y Stercorarius pomarinus (Temminck, 1815), spreading from the Ecuadorian coast to the extreme south, where they have been recorded more frequently. Since 1935 up to now, only thirteen articles registering the presence of any of these species in the extreme south of Chile have been released. Publications on behavior, ecology and phylogeny of these birds are scarce, and they are referred to investigations in Argentina and the northern hemisphere.
\end{abstract}

Keywords: Stercorariidae, Skuas, Extreme south in Southern Chile, State of knowledge.

\section{RESUMEN}

Los salteadores chilenos pertenecen a una familia relativamente poco estudiada y las investigaciones del grupo comprenden mayoritariamente avistamientos durante expediciones sobre la avifauna en el extremo sur de Chile. Reciben el nombre de "salteadores" por sus costumbres oportunistas; generalmente, se describen como gaviotas grandes, agresivas y carroñeras. El término "skua" proviene del

1 Departamento de Zoología, Universidad de Concepción, Casilla 160-C, Concepción, Chile. risoto@udec.cl 
feroés "skügver" que quiere decir "mechon de plumas" y de la isla Skúvoy del Archipiélago de las islas Feroe, en el Mar de Noruega.

En Chile existen seis especies: Stercorarius chilensis Bonaparte, 1957; Stercorarius longicaudus Vieillot, 1819; Stercorarius antarcticus (Lesson, 1831); Stercorarius maccormicki (Saunders, 1893); Stercorarius parasiticus (Linneo, 1758) y Stercorarius pomarinus (Temminck, 1815), distribuidas desde las costas ecuatorianas hasta el extremo sur, donde han sido registradas con mayor frecuencia. Desde 1935 a la fecha, solamente se han publicado quince artículos que registran la presencia de algunas de estas especies en el extremo sur de Chile. Publicaciones sobre comportamiento, ecología y Filogenia para estas aves son escasas y, están referidas a investigaciones en Argentina y en el hemisferio norte.

Palabras clave: Stercorariidae, Skuas, Extremo sur de Chile, Estado del conocimiento.

\section{INTRODUCCIÓN}

Chile cuenta con una nutrida avifauna que comprende 473 especies, (Vilina \& Pizarro, 2006), de las cuales 59 han sido alguna vez citadas para la Patagonia. Dentro de éstas, la familia Stercorariidae es un grupo de aves que posee seis especies descritas para Chile (Martínez \& González, 2004) de las nueve descritas a nivel mundial (Cohen et al. 1997). Se caracterizan por sus hábitos costeros y pelágicos (Narosky \& Babarskas, 2001).

Bullock (1935) las describe como "gaviotas grandes", con las alas más anchas y no tan largas, el color parecido al de Vanellus chilensis Molina, 1782 , pico robusto y terminado en gancho como las rapaces. El nombre de "salteador" proviene de las costumbres oportunistas, acosando a otras aves para quitarles el alimento (Bullock, 1935), pudiendo alcanzar velocidades de hasta $15 \mathrm{~m} / \mathrm{s}$ (Alerstam et al. 2007)

Dentro de los hábitos alimentarios de los adultos destacan el ser principalmente carroñeros, sin embargo, dentro de su dieta se encuentran peces, moluscos, crustáceos (Votier et al. 2003, Gutzwiller $2010^{1}$ ) y polluelos de otras aves. Stercocarius skua lonnbergi (Mathews, 1912) en Nueva Zelandia, caza polluelos de petreles durante la noche (Young et al. 1988), comportamiento similar al observado en Stercorarius antarcticus (Lesson, 1831) al alimentarse de pingüinos (Lazo et al. 1990).

1 Gutzwiller, S. 2010. Postcranial skeletal pneumaticity, bone stucture, and foraging style in two clades of neognath birds. Thesis Presented to The Honor Tutorial College, for the degree of Bachelors of Science in Biological Sciences, Ohio University. $72 \mathrm{pp}$.
A pesar que la crianza cooperativa se restringe mayoritariamente a aves terrestres, Stercorariidae parece ser la excepción, así lo explica Hemming (1994) señalando que si bien la mayoría de las aves marinas presentan monogamia, en el caso de los salteadores, pueden generar tríos de aves adultas dispuestas a defender el nido en forma conjunta, no obstante, este comportamiento en el hemisferio sur sólo estaría restringido a Stercorarius antarcticus.

La filogenia del grupo ha sido una gran controversia, Cohen et al. (1997) sugiere en base a la evidencia molecular y morfológica que Stercorariidae es un grupo monofilético, indicando que excepcionalmente Stercorarirus pomarinus, presenta ciertas características morfológicas y conductuales intermedias entre los géneros Stercorarirus y Catharacta, encontrándose más vinculado con el segundo género que con el primero, exclusivo del hemisferio sur. Es más, señala que de las seis especies de salteadores registrados en Chile, tres se encuentran emparentadas, mientras que las otras dos se encuentran en clados completamente diferentes y distantes. Por otro lado, Braun \& Brummfield (1998), ponen en duda la hipótesis de Cohen, señalando que la problemática que existe en el grupo recae sobre $S$. pomarinus, el cual debe ser considerado como un grupo hermano del Género Catharacta, incluso podría considerarse como un nuevo género. Actualmente, algunos autores consideran a $S$. antarcticus, $S$. lonnbergi y $S$. hamiltoni como subespecies de $S$. antarcticus generando ejemplares híbridos con características intermedias, producto de la cruza entre las distintas especies de Stercorarius (Ledesma et al. 2004).

Esta revisión tiene como objetivo mostrar la historia y el estado de conocimiento que existe de 
esta familia en el extremo sur de Chile que es donde más registros se han documentado.

\section{MATERIAL Y MÉTODOS}

Se realizó una búsqueda exhaustiva de las publicaciones entre los años 1920 al 2011 tanto de revistas, boletines y noticiarios referentes a la avifauna asociada al extremo sur de Chile y que contenían información sobre la familia Stercorariidae. De la información recabada se extrajo la historia de la familia para el sur de Chile.

La clave de identificación fue realizada mediante el uso de material fotográfico proporcionado por los fotógrafos Trevor Hardaker, Jari Peltomäki, Örn Óskarsson, Rodrigo Reyes y Pablo Cáceres. Además se revisó el material de la colección del Museo de Zoología (MZUC-UCCC) del Departamento de Zoología, Facultad de Ciencias Naturales y Oceanográficas de la Universidad de Concepción, de donde se obtuvo la mayor parte de las características para la confección de dicha clave. Para la distribución se siguió la sugerida por The Global Register of Migratory Species (2004) y los manuales de campo de Araya \& Millie (1989) y Martínez \& González (2004).

\section{RESULTADOS}

Historia de la familia Stercorariidae en el sur de Chile

En Chile el registro de esta familia se inicia con el trabajo de Bullock (1935), quien realiza una revisión de los avistamientos hechos por el Dr. Charles E. Hellmayr en 1932, quien registra el anidamiento de estas aves en Tierra del Fuego, señalando como único lugar definido de anidación es en la isla Santa María en el golfo de Arauco, aunque encontró huevos en Punta Angamos, bahía de Mejillones, Antofagasta. Posteriormente, Philippi (1959) realiza una revisión de la familia, detallando costumbres alimentarias, de nidificación y realizando una comparación entre las especies encontradas hasta ese momento. Así mismo, menciona que Stercorariidae posee sólo cuatro especies, y una con cinco subespecies. En cuanto a la distribución, Philippi afirma que pueden encontrarse en toda la costa chilena, especialmente en el extremo sur, desde Magallanes, Tierra del Fuego y la Antártica. Barros (1971) registra el avistamiento de Stercorarius chilensis en noviembre de 1968, desde las rocas de Eugenia hasta el islote Snipe y por el Beagle hasta Banner en Picton (Cabo de Hornos). Las aves se encontraban cazando gaviotines, comportamiento similar fue observado desde una avión dos días antes entre caleta Pescado y caleta Carlos, sobre el canal Beagle, en isla Nueva, volando a unos doscientos metros de altura.

Jory et al. (1974) registran la presencia de Stercorarius chilensis en el Parque Nacional "Laguna de los Cisnes" en Tierra del Fuego como residente de verano (anidación) pero no de invierno, aún así la consideran como habitante permanente de la Región de Magallanes. Además, documentan por primera vez la preferencia de estas aves por anidar sobre espacios abiertos y expuestos; las mayores concentraciones se encontraron en las mesetas altas y descubiertas de las islas dentro de la laguna. Venegas (1976), realiza observaciones ornitológicas en cinco estaciones ubicadas en Magallanes, bahía Stony, bsla Piazzi, bsla Rennell, isla Vidal Gormaz e isla Virtudes, registrando cuatro, dos, tres, tres y ningún ejemplar de $S$. chilensis para cada estación, respectivamente. Cinco años después Venegas (1981) registra la presencia de $S$. chilensis para las islas Wollaston y Bayly, con dos y tres ejemplares, respectivamente. Cabe señalar que Guzmán et al. (1986), realizaron un trabajo en verano sobre las mismas estaciones de Venegas (1976), y no encontraron salteadores. Lazo (1990) realizó una detallada investigación sobre los hábitos alimentarios de $S$. antarcticus, en la península de Byer, isla Livingston. Los censos realizados en 1988 determinan el estrecho vínculo que existe entre esta especie de salteador con Oceanites oceanicus Kuhl, 1820 y Fregetta tropica (Gould, 1844) formando parte del $40 \%$ de la dieta de $S$. antarcticus tanto de carroña como de presa. En sus investigaciones, concluyen que la baja densidad en la península en comparación con otras islas cercanas, donde las parejas nidificantes estaban a $30 \mathrm{~m}$ unas de otras, estaría explicada por la falta de colonias de pingüinos. Venegas (1991) documenta la observación de S. chilensis en el archipiélago Cabo de Hornos, sobre las islas Grevy, Bayly, Wollaston y Deceit, sin hacer referencia a comportamiento ni a otros hábitos. Venegas (1994), en su trabajo sobre la isla Noir, no incluye a $S$. chilensis dentro de sus resultados por tratarse de un ave pelágica, a pesar 
de estar presente en todos los censos sobrevolando a gran altura. El mismo autor para el año 1995 registra las observaciones de siete individuos de $S$. chilensis en las inmediaciones del aeropuerto de Punta Arenas, todas ellas entre diciembre y febrero (Venegas, 1995). Imberti (2005) en su trabajo sobre los canales chilenos, observa a $S$. pomarinus en el estero Hito y $S$. chilensis entre el estero Hito y Castro. Por último y muy posteriormente, Garay et al. (2008), señalan la presencia de Stercorarius chilensis en el fiordo Última Esperanza, y no se detallan el punto de observación donde la especie fue registrada.

Diagnosis y Clave de Identificación de Campo

A nivel mundial se conocen nueve especies de salteadores, de las cuales seis son habituales en Chile, sobre todo de la Región Patagónica y Antártica

A continuación, se muestra la diagnosis de identificación de estas aves, junto con su distribución y una clave de identificación de campo. Para la compresión de los colores e identificación del mismo, téngase presente la diferencia entre "pardo" y "café" y sus derivados. Primeramente, el color "pardo" alude a tonalidades rojas a amarillos anaranjadas, propias del pelaje del leopardo (Panthera pardus Linnaeus, 1758) o el oso pardo (Ursus arctos Linnaeus, 1758). Por otro lado, el color café es basado en la tonalidad de la bebida del mismo nombre.

\section{familia Stercorariidae (Laridae: Charadriiformes)}

Stercorarius chilensis Bonaparte, 1957: L. 55/61. Partes superiores con una coloración café grisácea, con pequeñas manchas blanquecinas y ocráceas. Cuello con estrías amarillentas o blanquecinas. Mancha blanca en las alas; pico y patas negros. Distribución: desde Perú hasta Magallanes y Tierra del Fuego, se le puede encontrar en la Patagonia argentina (Araya \& Millie, 1989) (Fig. 1, 11). Monotípica, marina pero no pelágica, vinculada a la costa, ocupando fiordos y canales australes (Martínez \& González, 2004).

Los adultos pueden ser encontrados en grupos pequeños de 5 a 8 individuos, el huevo es de forma oval de color verde oliva como color base y manchados irregularmente en varios tonos pardo, sobre un nido hecho de restos vegetales de junquillo (Kush et al. 2007).

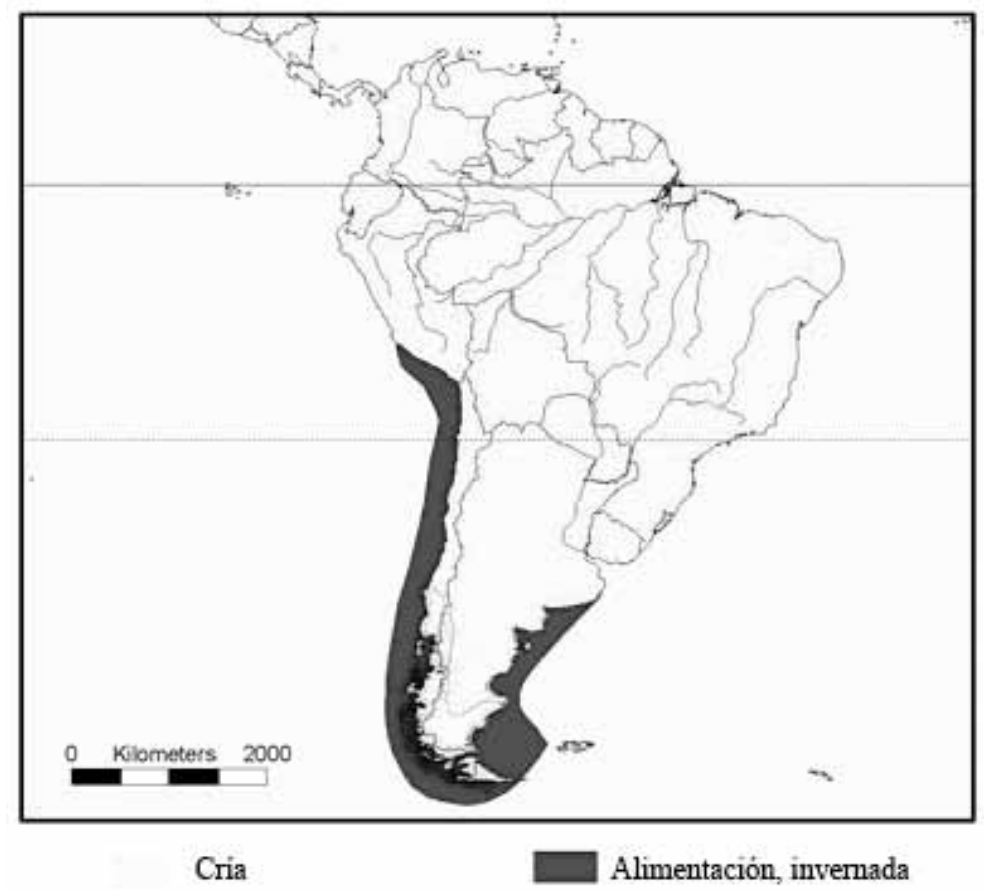

Fig. 1. Distribución de Stercorarius chilensis. Modificado de: The Global Register of Migratory Species (www.groms.de). 


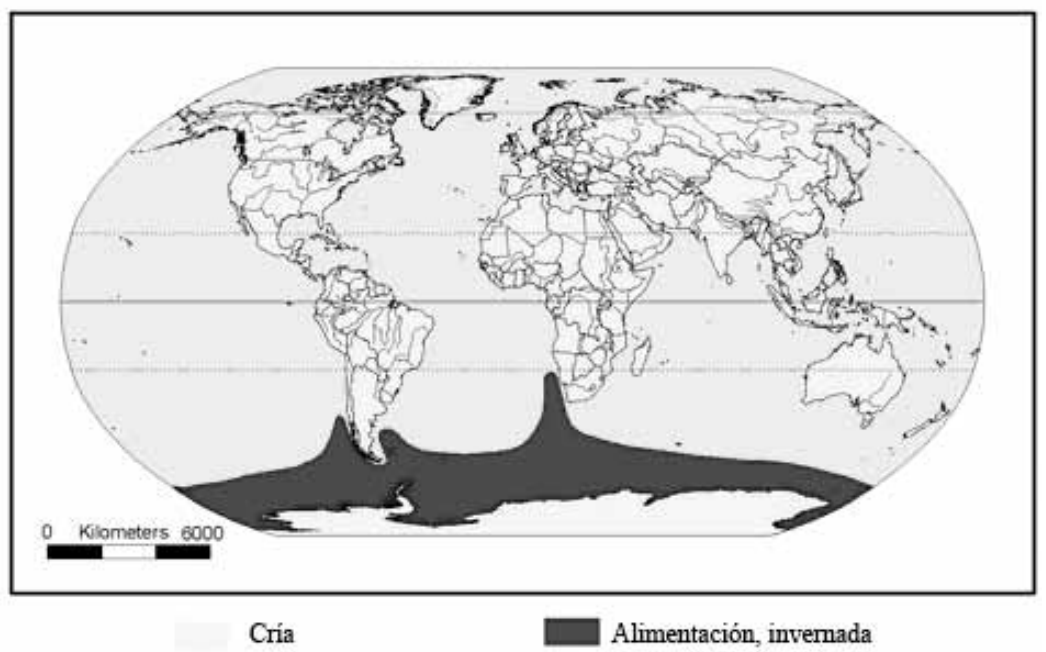

Fig. 2. Distribución de Stercorarius longicaudus. Modificado de: The Global Register of Migratory Species (www.groms.de).

Stercorarius longicaudus Vieillot, 1819: L. $48 / 58 \mathrm{~cm}$. Partes superiores de color grisáceo pálido. Carece de banda pectoral. Las rectrices centrales son más largas, sobresaliendo bastante del resto de la cola, mucho más que otras especies. Fase oscura es rara. Difícil de distinguir entre Stercorarius parasiticus, ya que en la época en que se encuentra en Chile las plumas centrales de la cola no están alargadas (Araya \& Millie, 1989). Ave marina y altamente pelágica fuera de su período de cría. Con dos subespecies aceptadas, se reproduce en el circulo polar ártico, migra al sur para el invierno boreal (Martínez \& González, 2004) (Fig. 2, 8).
Stercorarius antarcticus (Lesson, 1831): L. $52 / 64 \mathrm{~cm}$. Coloración parda negruzca en las partes superiores del cuerpo y alas. Manchas pálidas en el manto. Partes inferiores con pardo más pálido. Pico y patas negras. La más grande del grupo. Muy parecida a $S$. chilensis. En vuelo con alas puntiagudas con manchas blancas en cada extremo. Cola cuneada. Tres subespecies reconocidas. En el extremo sur de Sudamérica y sus islas (Martínez \& González, 2004) (Fig. 3, 12.A).

Stercorarius maccormicki (Saunders, 1893): Longitud de $53 \mathrm{~cm}$. Con dos fases de coloración, una clara y otra oscura. La primera con las partes

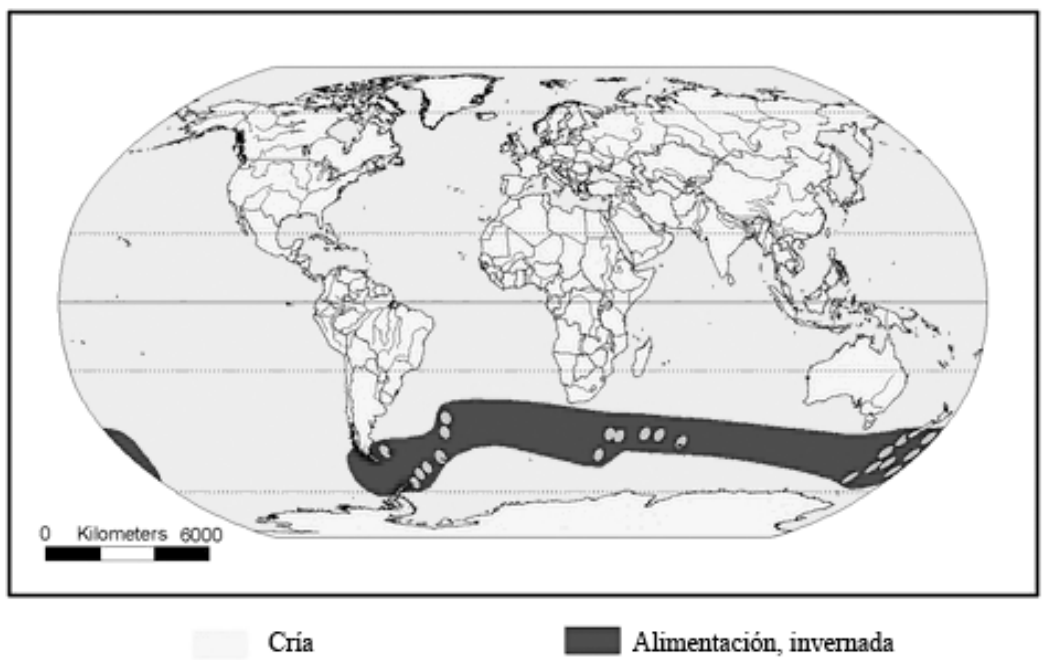

Fig. 3. Distribucion de Stercorarius antarcticus. Modificado de: The Global Register of Migratory Species (www.groms.de). 


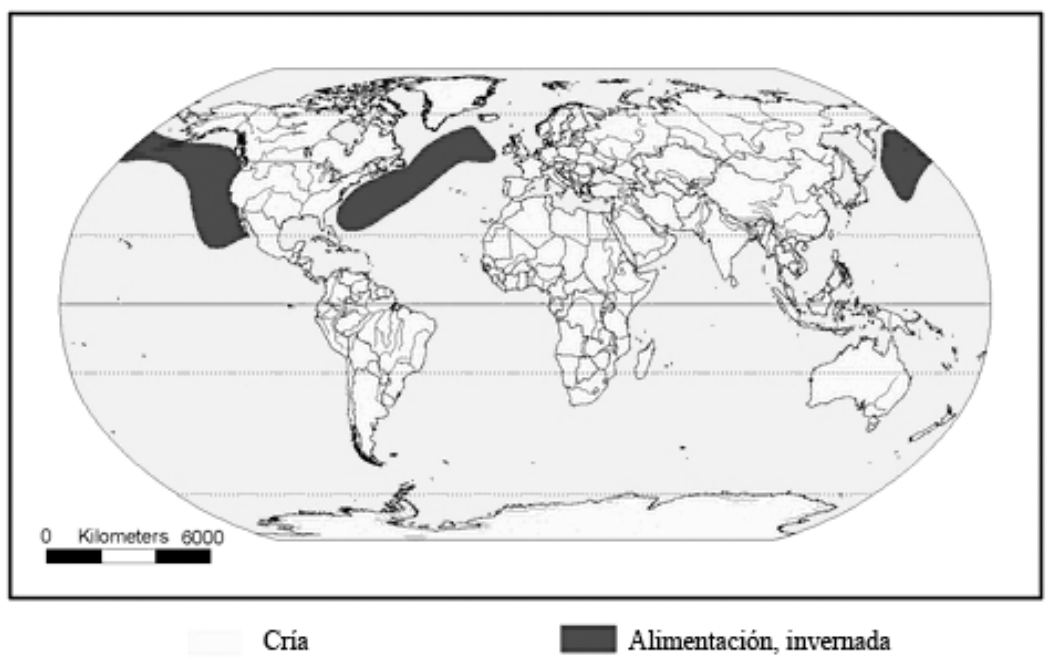

Fig. 4 Distribución de Stercorarius maccormicki. Extraido de: The Global Register of Migratory Species (www.groms.de).

superiores parda oscuras y las inferiores gris pardas pálidas con estrías doradas prominentes en el cuello. En la fase oscura el dorso es igual a la fase clara, sin embargo las partes inferiores son más obscuras de color pardo, con las plumas doradas del cuello menos prominentes. Ambas fases con mancha blanca en las alas. Pico y patas negros, a veces con algo de blanco (Araya \& Millie, 1989). Monotípico, se distribuye en la Antártica donde se reproduce, durante el inverno austral migran hacia el hemisferio norte siguiendo la corriente de Humboldt (González \& Martínez, 2004) (Fig. $4,10$ y $12 . B)$.
Stercorarius parasiticus (Linneo, 1758): L. $41 / 50 \mathrm{~cm}$. Región loral, frente y corona de color pardo grisáceo, cuello blanco, resto de la superficie dorsal gris pardusca, incluidas las cobertoras alares, rémiges y rectrices pardo oscuras, las rémiges con raquis blancos. Superficie ventral: lados de la cara y cuello blanco bañado de amarillento; garganta y todo el resto ventral blanco excepto las cobertoras subcaudales y subalares, gris parduscas (fase clara). Cuello y superficie ventral parda grisácea o ahumado, sólo ligeramente más claro que la superficie dorsal y algunas veces tintes amarillentos a los lados del cuello (fase oscura). Pico de color claro con punta

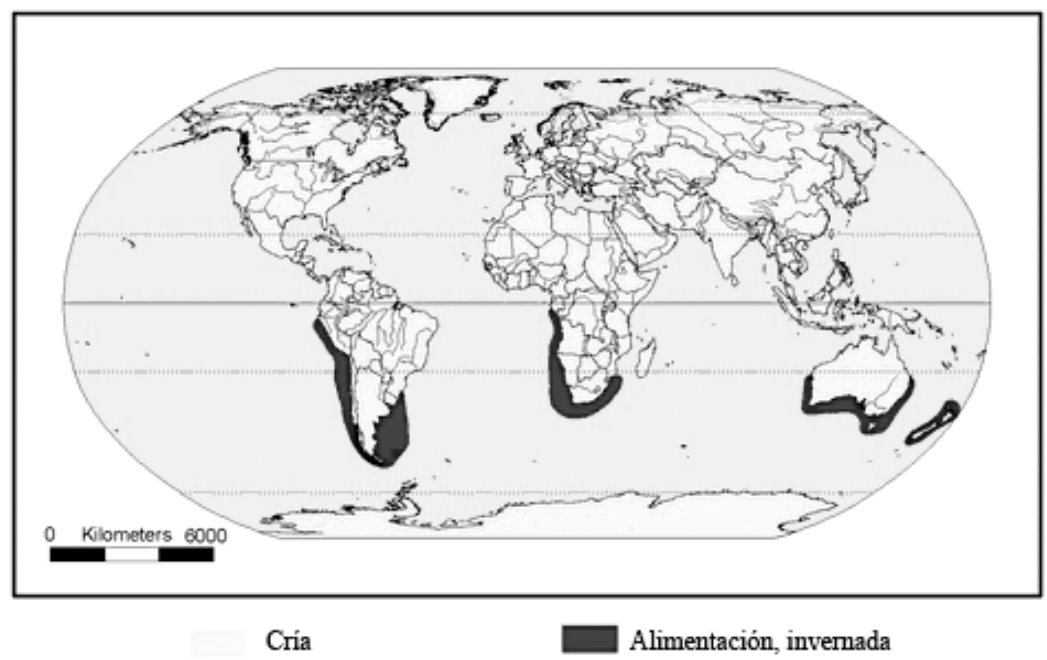

Fig. 5. Distribución de Stercorarius parasiticus. Extraido de: The Global Register of Migratory Species (www.groms.de). 


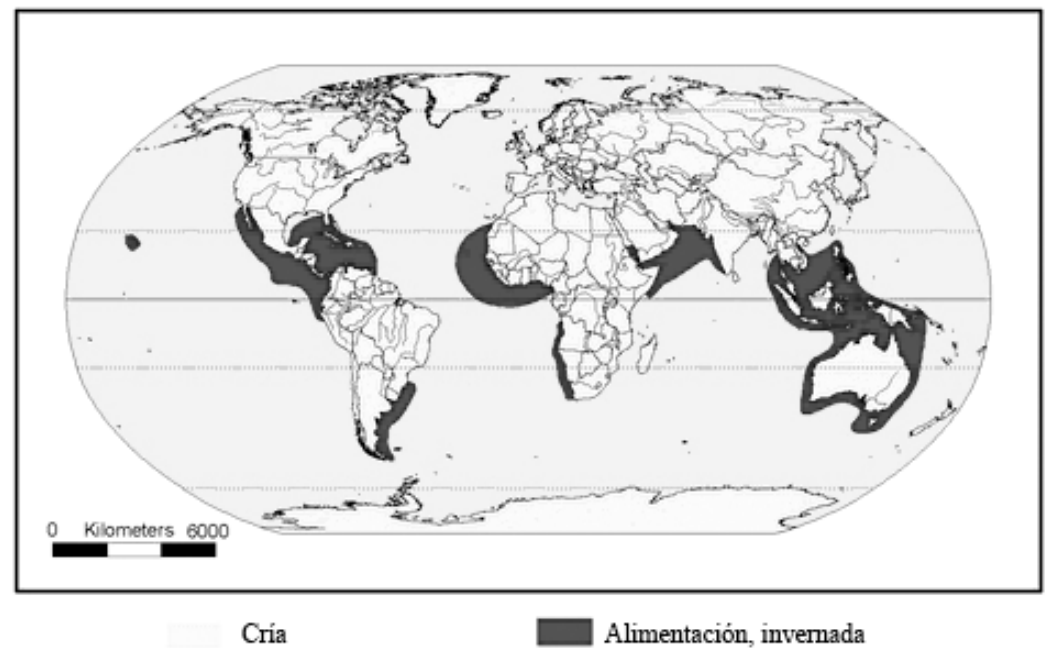

Fig. 6. Distribución de Stercorarius pomarinus. Extraido de: The Global Register of Migratory Species (www.groms.de).

oscura. Tarsos y dedos negros. Cola larga y cunea$\mathrm{da}$, con las dos rectrices centrales puntiagudas y sobresalientes del resto de la cola. Iris pardo (Araya \& Millie, 1989). Monotípico. Desde Ecuador hasta el Pacífico sur, Chile y Argentina, migra hacia el sur durante el invierno boreal (Martinez \& González 2004) (Fig. 5, 9).

Stercorarius pomarinus (Temminck, 1815): L. 48/58 cm. Plumas centrales de la cola más largas torcidas y con borde redondeado. Con dos fases de coloración, la fase oscura en donde el ave es completamente gris oscuro. Por otro lado, en la fase clara, la cara y las partes inferiores son blancas

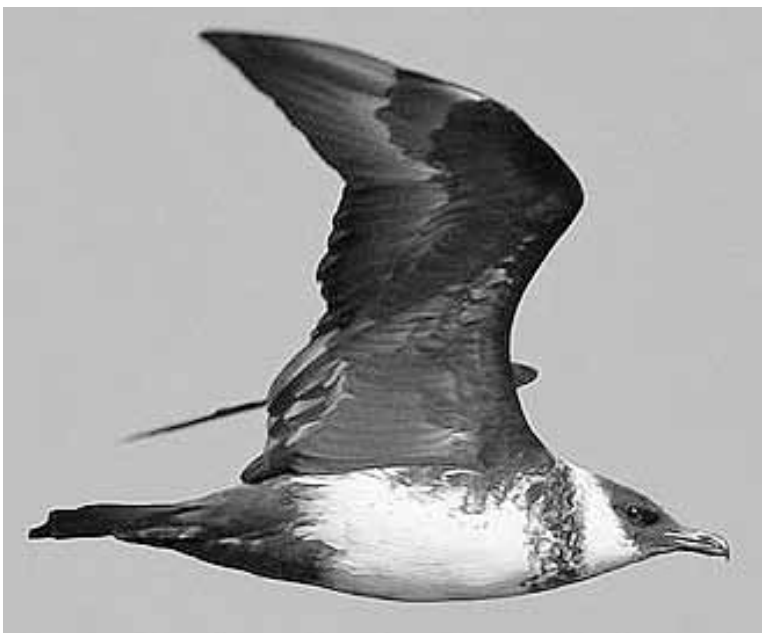

Fig. 7. Stercorarius pomarinus (C) Trevor Hardaker Wild Life Photographer 2008 y la banda pectoral y cara inferior de las alas son café oscuro. En ambas fases la corona es negra con pico amarillento oscuro y las patas negras (Araya \& Millie, 1989). Monotípico, migra al sur durante el invierno boreal, desde Perú hasta el extremo austral de Chile y Argentina (Martínez \& González, 2004) (Fig. 6, 7).

Clave para la identificación de las especies de la familia Stercorariidae

1.- Nuca con collar amarillento. 2

Nuca sin collar amarillento. 4

2.- $\quad$ Primarias con color blanco en el raquis más externo, vientre más pálido que el resto del cuerpo........................................................................

Primarias sin color blanco en el raquis más externo, vientre blanco.. Stercorarius pomarinus (Fig. 7)

3.- $\quad$ Rectrices centrales más largas que las del resto de la cola. Sin mostacho, collar del pecho completo y visible...Stercorarius longicaudus (Fig. 8)

Cola cuneada con centrales más largas. Collar del pecho difuso centralmente, con mostacho........................ Stercorarius parasiticus (Fig. 9)

4.- $\quad$ Con dos fases de coloración...................5

Con una fase de coloración..................6 


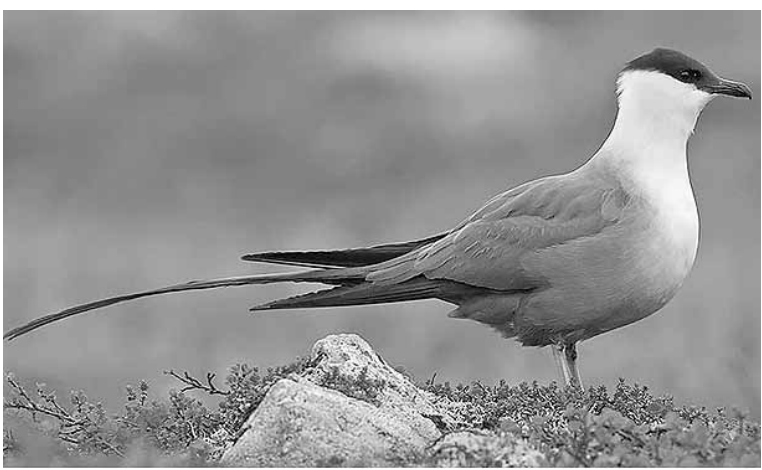

Fig. 8. Stercorarius longicaudus (c) Jari Peltomäki. International Bird Photographer 2010

5.- $\quad$ Completamente pardo oscuro, levemente pálido en el abdomen. .............. Stercorarius maccormicki en fase oscura (Fig. 10)

Pardo negruzco, vientre cabeza y pecho pardo pálido. Stercorarius maccormicki en fase clara (Fig. 12.B)

6.- $\quad$ Manto y alas color pardo negruzcas sin manchas. Vientre y pecho pardo rojizo. Stercorarius chilensis (Fig. 11)

Manto y alas color pardo negruzcas con manchas blancas o pálidas. Vientre y pecho más pálidos que el resto del cuerpo. Coloración parda rojiza sólo en la nuca....

Stercorarius antarcticus (Fig. 12.A)

\section{DISCUSIÓN Y CONCLUSIONES}

Seis especies de la familia Stercorariidae han sido descritas para Chile, y dentro las quince publicaciones que las refieren al país sólo se documenta a $S$. chilensis, $S$. antarcticus y $S$. pomarinus por lo que $S$. maccormicki, $S$. parasiticus y $S$. longicaudus se consideran en esta revisión por encontrarse incluidas dentro de los manuales de campo de Aralla \& Millie (1989) y Martínez \& González (2004).

Debido a que la familia posee una alta capacidad de hibridación, (Pierotti \& Annett, 1993), resulta difícil generar una identificación clara de los especímenes que se registran, y aunque estos híbridos sólo han sido registrados en Argentina (Ledesma et al. 2004) y en la Antártica (Andersson, 1999), no significa que puedan estar presentes en otras áreas del territorio chileno, de manera que no se descarta

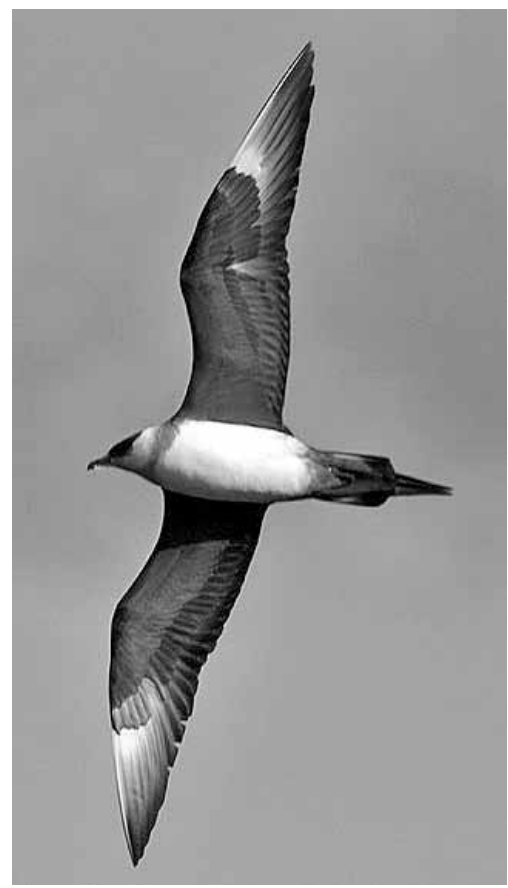

Fig. 9. Stercorarios parasiticus (C) Örn Óskarsson Teacher of Biology Photographer 2009

la hibridación de las especies, situación que no ha sido estudiada en el país.

El grueso de los documentos citados en los resultados, hacen referencia a la presencia de la familia, pero los registros corresponden a meras observaciones sobre avifauna en general de regiones australes, existiendo un vacío de información, sobre

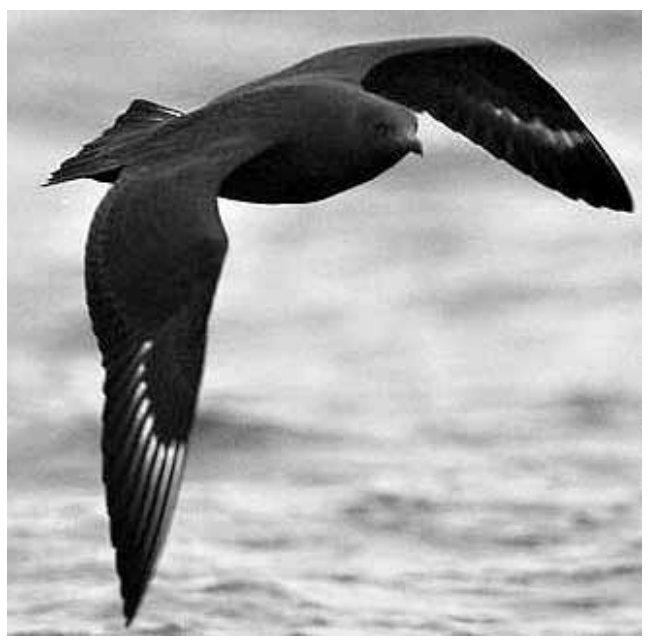

Fig. 10. Stercorarius maccormicki en fase oscura (C) Rodrigo Reyes Aceitón 2005 


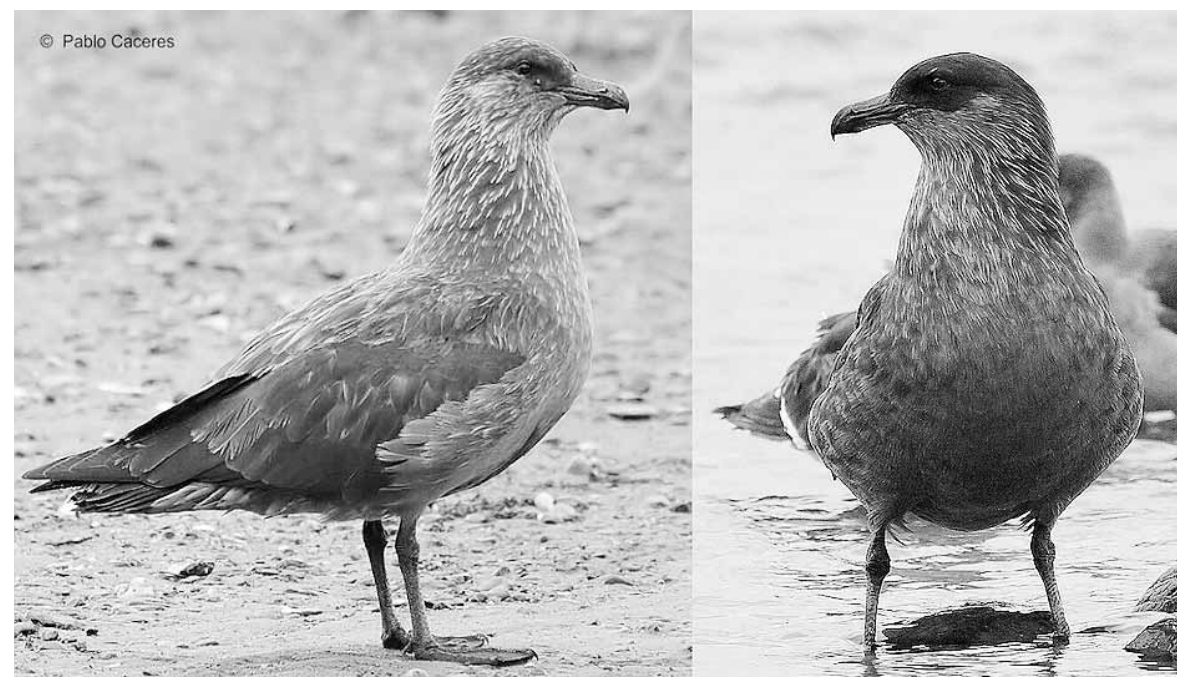

Fig. 11. Stercorarius chilensis (c) Pablo Cáceres Contreras Fotógrafo 2008.

todo en disciplinas como filogenia, ecología y etología, así como patrones de distribución de la familia en Chile, particularmente en el extremo sur. A pesar de esto, las escasas, investigaciones sobre su ecología y comportamiento (Bullock, 1935, Philippi, 1959, Barros 1971, Lazo 1990), aluden a la importancia que posee la familia sobre otras aves, controlando el tamaño poblacional de éstas. Dichos artículos se restringen sólo a $S$. antarcticus, excepto el de Philippi (1959), quien relata la actividad de alimentación de la familia en general y no precisa de cuáles especies se trata. Documentar el comportamiento de estas aves y su relación ecológica con otras, aportaría datos importantes para distinguir hibridación, que como dice Ledesma et al. (2004), poseen características intermedias entre cada especie.

En cuanto a distribución son preferentemente de regiones extremas, realizando la cría en el hemisferio norte como $S$. longicaudus, $S$. parasiticus y $S$. pomarinus, otros como $S$. antarcticus y $S$. chilensis son exclusivamente del hemisferio sur. La distribución real de la familia en el extremo sur de Chile aún sigue siendo desconocida, señalándose con propiedad la presencia de las tres especies

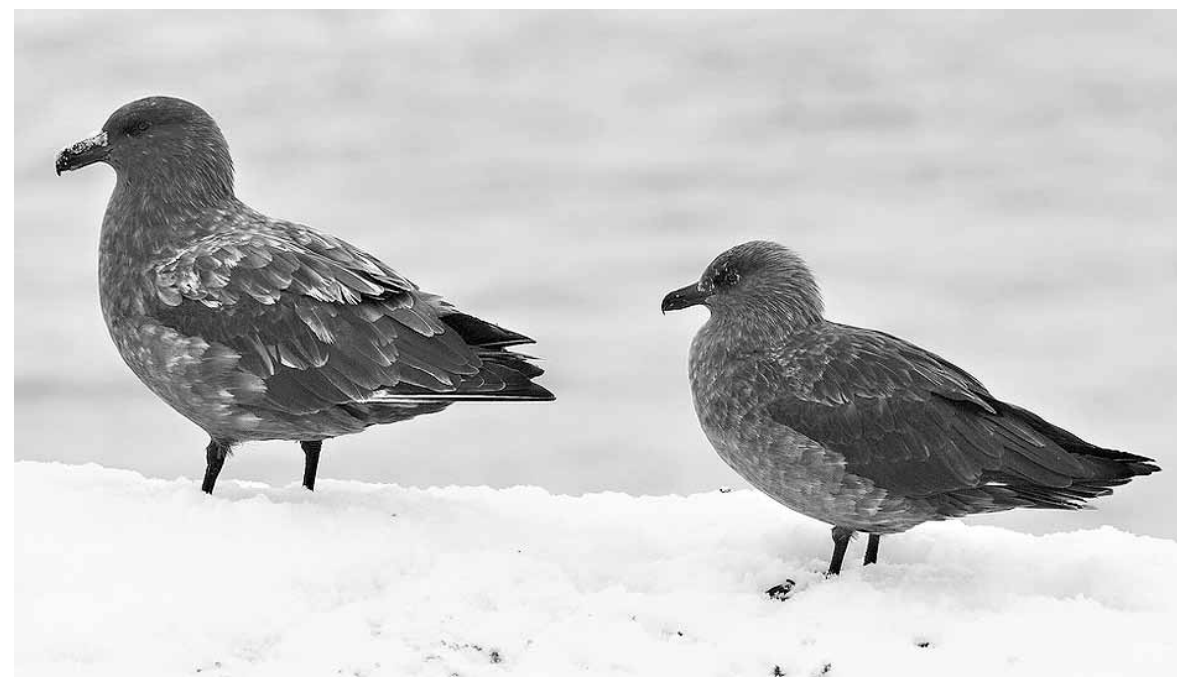

Fig. 12. Stercorarius antaritus (A) y Stercorarius maccormicki (B) en su fase clara C Pablo Cáceres Contreras Fotógrafo 2008. 
antes mencionadas (S. chilensis, S. antarcticus y $S$. pomarinus) en los artículos citados.

Los manuales de campo consultados aportan una valiosa referencia para la identificación de estas aves, no obstante, en el trabajo de campo los datos obtenidos dependen de: la distancia que hay entre el ave y el observador, luminosidad, clima, estación del año y de la experiencia del observador entre otras cosas, como del equipo que se dispone. Bajo este marco, la clave realizada genera un instrumento útil y sencillo para la identificación de las especies listadas, sobre todo las que poseen más de un tipo de plumaje al año.

Finalmente, se expone de esta manera, a pesar de la dificultad que presenta la familia en sí, la necesidad inmediata de rellenar los vacíos que existen en cuando al conocimiento sobre ésta y las potenciales líneas de investigación que se pueden generar.

\section{AGRADECIMIENTOS}

Agradecemos especialmente a los fotógrafos Pablo Cáceres, Rodrigo Reyes Aceitón, Örn Óskarsson, Jari Peltomäki, por su colaboración al entregarnos las autorizaciones sobre sus fotografías incluidas en el presente escrito.

\section{LITERATURA CITADA}

Alerstam, T., M. Rosén, J. Bäckman, P. Ericson \& O. Hellgren 2007 Fligth Speeds among Birds Species: Allometric and Philogenetic Effects. PL Biology, 5(8): 1656-1662.

Andersson, M. 1999. Hybridization and skua phylogeny. Proceedings of the Royal Society Biological Sciences, 266: 1579-1585.

Araya, B. \& G. Millie 1989 Guía de campo de las aves de Chile. Editorial Universitaria. 406 pp.

Barros, A. 1971. Aves Observadas en las Islas Picton, Nueva, Lennox y Navarino Oriental. Anales del Instituto de la Patagonia, 2(1-2): 166-180.

Braun, M. \& R. Brumfield 1998. Enigmatic phylogeny of skuas: an alternative hypotesis. Proceedings of the Royal Society Biological Sciences, 265: 995-999.

Bullock, D. 1935. El Peuco del Mar o "Salteador". Revista Chilena de Historia Natural, 39. 169-171.
Cohen, B., A. Baker, K. Blechschmidt, D. Dittmann, R. Furness, J. Gerwin, A. Helbig, J. de Korte, H. Marshall, R. Palma, H. Petter, R. Ramli, I. Siebold, M. Willcox, R. Wilson \& R. Zink 1997. Enigmatic phylogeny of skuas (Aves: Stercorariidae). Proceedings of the Royal Society Biological Sciences, 264 (1379): 181-190.

Garay, G., O. Guineo, E. Mutschke \& C. Ríos 2008. Tamaño, estructura y distribución estacional de poblaciones de aves acuáticas en el Fiordo Última Esperanza y Canal Señoret, Región de Magallanes. Anales del Instituto de la Patagonia, 36(2): 33-34.

Guzmán, L., A. Atalah \& C. Venegas 1986. Composición específica y estructura de la comunidad de aves de verano en el complejo de la Tundra Magallánica. Anales del Instituto de la Patagonia, 16: 75-86.

Hemming, A. 1994. Cooperative breeding in the skuas (Stercorariidae): history, distribution and incidence. Journal of The Royal Society of the New Zealand, 24(3): 245-260.

Imberti, S. 2005. Distribución otoñal de las aves marinas y terrestres en los canales chilenos. Anales del Instituto de la Patagonia, 33: 21-30.

Jory, J., C. Venegas \& A. Texera 1974. La avifauna del Parque Nacional "Laguna de los Cisnes" Tierra del Fuego, Chile. Anales del Instituto de la Patagonia, 5(1-2): 131-154.

Kusch, A., M. Marín, D. Oheler \& S. Drieschman 2007. Notas sobre la avifauna de la Isla Noir

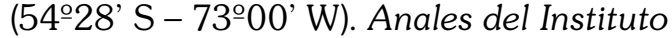
de la Patagonia, 35(2): 61-66.

Lazo, I., P. Cattan, H. Nuñez \& J. Yáñez 1990. Hábitos alimentarios del salteador pardo (Catharacta lonnbergi) en ausencia de colonias de pingüinos, Isla Livingston, Shetland del sur. Serie Científica INACH, 40: 71-77.

Ledesma, M., R. Gunski, D. Montalti \& G. Leotta 2004. Estudios citogenéticos en skuas (Charadriiformes) y sus productos híbridos. Actas del $\mathrm{VI}^{\circ}$ Simposio Argentino y $\mathrm{III}^{\circ}$ Latinoamericano sobre Investigaciones Antárticas CD-ROM. Comunicaciones № 220BA. 1pp.

Martínez, D. \& G. González 2004. Las Aves de Chile, Nueva guía de campo. Ediciones del Naturalista. Santiago, Chile. 620 pp. 
Narosky, T. \& M. Babarskas 2001. Aves de la Patagonia, guía para su reconocimiento. Vázquez Mazzini Editores. Buenos Aires. Argentina. 128 pp.

Philippi, R. 1959. Las Gaviotas Salteadoras. Noticiario Mensual del Museo Nacional de Historia Natural, 3 (31): 5-7.

Pierotti, R. \& C. Annett 1993. Hybridization and male parental investment in birds. The Condor, 95: 670-679.

The Global Register of Migratory Species. 2004. www.groms.de

Venegas, C. 1976. Observaciones Ornitológicas en la tundra Magallánica. Anales del Instituto de la Patagonia, 7: 171-184.

Venegas, C. 1981. Aves de las Islas Wollaston y Bayly, Archipiélago del Cabo de Hornos. Anales del Instituto de la Patagonia, 12: 13-19.

Venegas, C. 1991. Ensambles avifaunísticos estivales del Archipiélago Cabo de Hornos. Anales del Instituto de la Patagonia, 20: 69-82.
Venegas, C. 1994. Comunidades de aves terrestres en Isla Noir. Anales del Instituto de la Patagonia, 22: 43-49.

Venegas, C. 1995. Variación diaria y estacional de la avifauna asociada al aeropuerto de Punta Arenas. Anales del Instituto de la Patagonia, 23: 35-45.

Vilina, Y. \& C. Pizarro 2006. Aves Marinas. In CONAMA (ed.). Biodiversidad de Chile. Patrimonio y Desafíos. Ocho Libros Editores (Santiago de Chile). 640 pp.

Votier, C., S. Bearhop, A. MacCormik, N. Ratcliffe \& W. Furness 2003. Assessing the diet of great skuas, Catharacta skua, using five different techniques. Polar Biology, 26: 20-26.

Young, E., P. Jenkins, E. Douglas \& T. Lovegrove 1988. Nocturnal foraging by Chatham Island Skuas. New Zeland Journal of Ecology, 11: 113-117. 
